\title{
BIOASSAY DATA FOR MARINE POLLUTION USING SEA URCHIN EGGS, 1974
}

AUTHOR(S):

Kobayashi, Naomasa

\section{CITATION:}

Kobayashi, Naomasa. BIOASSAY DATA FOR MARINE POLLUTION USING SEA URCHIN EGGS, 1974. PUBLICATIONS OF THE SETO MARINE BIOLOGICAL LABORATORY 1976, 22(6): 405-407

ISSUE DATE:

1976-02-28

URL:

http://hdl.handle.net/2433/175909

RIGHT: 


\section{BIOASSAY DATA FOR MARINE POLLUTION USING SEA URGHIN EGGS, 1974}

In 1974, five experiments for biological assay were made with sea urchin eggs to measure marine pollution around the Seto Marine Biological Laboratory.

I. Spring season: An experiment was made on March 14, with Hemicentrotus pulcherrimus (A. Agassiz) eggs, see Table 1.

II. Summer season: Two experiments were made in June-August, with Anthocidaris crassispina (A. Agassiz) eggs. For Exp. 1 on June 9 see Table 2, and for Exp. 2 on August 6 see Table 3.

III. Autumn season: Two experiments were made in September-November, with Anthocidaris crassispina or Pseudocentrotus depressus (A. Agassiz) eggs. For Exp. 1 on September 14 see Table 4, and for Exp. 2 on November 27 see Table 5.

(Notes common to all tables: in Fertilization the membrane formation was checked 3 minutes after insemination; minutes and hours in parentheses respectively after First cleavage and Gastrulation indicate the time after insemination; the maturation state of gonads used was nearly to full ripe and eggs were experimented with after

Table 1. Results of the Mar. 14 experiment with eggs of Hemicentrotus pulcherrimus. Wind; NW1. Test water temperature; $19^{\circ} \mathrm{C}$. 6 hrs. old eggs

\begin{tabular}{|c|c|c|c|c|c|c|c|c|c|}
\hline \multirow{2}{*}{$\begin{array}{c}\text { Location } \\
\text { (depth) }\end{array}$} & \multirow{2}{*}{\begin{tabular}{|l|} 
Fertiliz. \\
$\begin{array}{l}\text { membrane } \\
\text { formation }\end{array}$ \\
\end{tabular}} & \multicolumn{3}{|c|}{ First cleavage ( $75 \mathrm{~min})}$. & \multicolumn{3}{|c|}{ Gastrulation (20 hrs.) } & \multirow{2}{*}{\begin{tabular}{|c|}
$\begin{array}{c}\text { Other } \\
\text { notes }\end{array}$ \\
ab- \\
normal \\
develop.
\end{tabular}} & \multirow{2}{*}{$\begin{array}{c}\text { Degrce } \\
\text { of in- } \\
\text { hibitory } \\
\text { effect }\end{array}$} \\
\hline & & 1 cell & $\mid \begin{array}{c}2 \text { cell } \\
\text { (normal) }\end{array}$ & $\begin{array}{c}\text { multi- } \\
\text { cell(poly- } \\
\text { spermy) }\end{array}$ & $\begin{array}{c}\text { perma- } \\
\text { nent } \\
\text { blastula }\end{array}$ & $\begin{array}{c}\text { gastrula } \\
\text { (normal) }\end{array}$ & $\begin{array}{c}\text { exo- } \\
\text { gastrula }\end{array}$ & & \\
\hline $\begin{array}{l}\text { Running }(\mathrm{m}) \\
\text { sea water of } \\
\text { Laboratory }\end{array}$ & $\begin{array}{l}98.0 \% \\
99.5 \\
94.5\end{array}$ & $\begin{array}{l}2.5 \% \\
3.5 \\
6.5\end{array}$ & $\begin{array}{l}97.5 \% \\
96.5 \\
93.5\end{array}$ & $\begin{array}{l}0 \\
0 \\
0 \\
0\end{array}$ & $1.0^{\%}$ & $99.0^{\%}$ & $0^{\%}$ & & 0 \\
\hline $\begin{array}{l}\text { Water from } \\
\text { open sea side } \\
\text { of Hatake- } \\
\text { jima Surface }\end{array}$ & $\begin{array}{l}99.0 \\
96.5 \\
94.5\end{array}$ & $\begin{array}{l}3.5 \\
4.5 \\
7.0\end{array}$ & $\begin{array}{l}96.5 \\
95.5 \\
93.0\end{array}$ & $\begin{array}{l}0 \\
0 \\
0\end{array}$ & 1.0 & 99.0 & 0 & & 0 \\
\hline $\begin{array}{l}\text { Water from } \\
\text { land side of } \\
\text { Hatakejima } \\
\text { Surface }\end{array}$ & $\begin{array}{l}75.0 \\
79.5 \\
73.5\end{array}$ & $\begin{array}{l}27.0 \\
24.5 \\
28.5\end{array}$ & $\begin{array}{l}73.0 \\
75.5 \\
71.5\end{array}$ & $\begin{array}{l}0 \\
0 \\
0\end{array}$ & 1.0 & 99.0 & 0 & & 2 \\
\hline Bottom (27) & $\begin{array}{l}80.0 \\
81.5 \\
71.5\end{array}$ & $\begin{array}{l}22.0 \\
20.5 \\
31.0\end{array}$ & $\begin{array}{l}78.0 \\
79.5 \\
69.0\end{array}$ & $\begin{array}{l}0 \\
0 \\
0\end{array}$ & 3.0 & 97.0 & 0 & & 3 \\
\hline $\begin{array}{l}\text { Sea water } \\
\text { from Tsuna- } \\
\text { shirazu cove } \\
\text { Surface } \\
\end{array}$ & $\begin{array}{l}74.0 \\
75.0 \\
71.5\end{array}$ & $\begin{array}{l}26.5 \\
27.5 \\
29.5\end{array}$ & $\begin{array}{l}73.5 \\
72.5 \\
70.5\end{array}$ & $\begin{array}{l}0 \\
0 \\
0\end{array}$ & 3.5 & 96.5 & 0 & & 2 \\
\hline Bottom (5) & $\begin{array}{l}68.5 \\
70.5 \\
63.0\end{array}$ & $\begin{array}{l}36.5 \\
31.5 \\
38.5\end{array}$ & $\begin{array}{l}63.5 \\
68.5 \\
61.5\end{array}$ & $\begin{array}{l}0 \\
0 \\
0\end{array}$ & 7.5 & 91.5 & 1.0 & & 3 \\
\hline
\end{tabular}

Publ. Seto Mar. Biol. Lab., XXII, 405-407, $1976 . \quad$ (Biological data 2) 
Table 2. Results of the June 9 experiment with eggs of Anthocidaris crassispina. Wind; 0 . Test water temperature; $22^{\circ} \mathrm{C}$. $4 \mathrm{hrs}$. old eggs

\begin{tabular}{|c|c|c|c|c|c|c|c|c|c|}
\hline \multirow{2}{*}{$\begin{array}{c}\text { Location } \\
\text { (depth) }\end{array}$} & \multirow{2}{*}{\begin{tabular}{l|} 
Fertiliz. \\
$\begin{array}{l}\text { membrane } \\
\text { formation }\end{array}$
\end{tabular}} & \multicolumn{3}{|c|}{ First cleavage ( $60 \mathrm{~min})}$. & \multicolumn{3}{|c|}{ Gastrulation (18 hrs.) } & \multirow{2}{*}{\begin{tabular}{|c|}
$\begin{array}{c}\text { Other } \\
\text { notes }\end{array}$ \\
ab- \\
normal \\
develop.
\end{tabular}} & \multirow{2}{*}{$\begin{array}{c}\text { Degree } \\
\text { of in- } \\
\text { hibitory } \\
\text { effect }\end{array}$} \\
\hline & & 1 cell & $\begin{array}{c}2 \text { cell } \\
\text { (normal) }\end{array}$ & $\begin{array}{c}\text { multi- } \\
\text { cell(poly- } \\
\text { spermy) }\end{array}$ & $\begin{array}{c}\text { perma- } \\
\text { nent } \\
\text { blastula }\end{array}$ & $\begin{array}{c}\text { gastrula } \\
\text { (normal) }\end{array}$ & $\begin{array}{c}\text { exo- } \\
\text { gastrula }\end{array}$ & & \\
\hline $\begin{array}{l}\text { Running }(\mathrm{m}) \\
\text { sea water of } \\
\text { Laboratory }\end{array}$ & $\begin{array}{l}98.5 \% \\
98.0 \\
99.0\end{array}$ & $\begin{array}{l}2.5 \% \\
3.0 \\
2.0\end{array}$ & $\begin{array}{l}97.5 \% \\
97.0 \\
98.0\end{array}$ & \begin{tabular}{ll|}
0 & $\%$ \\
0 & \\
0 &
\end{tabular} & $0.5^{\%}$ & $99.5^{\%}$ & $\%$ & & 0 \\
\hline $\begin{array}{l}\text { Water from } \\
\text { open sea side } \\
\text { of Hatake- } \\
\text { jima Surface }\end{array}$ & $\begin{array}{l}96.5 \\
97.5 \\
98.0\end{array}$ & $\begin{array}{l}5.0 \\
3.5 \\
3.0\end{array}$ & $\begin{array}{l}95.0 \\
96.5 \\
97.0\end{array}$ & $\begin{array}{l}0 \\
0 \\
0\end{array}$ & 1.5 & 98.5 & 0 & & 0 \\
\hline $\begin{array}{l}\text { Water from } \\
\text { land side of } \\
\text { Hatakejima } \\
\text { Surface }\end{array}$ & $\begin{array}{l}95.5 \\
97.0 \\
97.5\end{array}$ & $\begin{array}{l}5.0 \\
3.5 \\
3.0\end{array}$ & $\begin{array}{l}95.0 \\
96.5 \\
97.0\end{array}$ & $\begin{array}{l}0 \\
0 \\
0\end{array}$ & 3.0 & 97.0 & 0 & & 0 \\
\hline Bottom (27) & $\begin{array}{l}95.0 \\
94.5 \\
93.0\end{array}$ & $\begin{array}{l}6.0 \\
7.0 \\
9.0\end{array}$ & $\begin{array}{l}94.0 \\
93.0 \\
91.0\end{array}$ & $\begin{array}{l}0 \\
0 \\
0\end{array}$ & 7.0 & 93.0 & 0 & & 1 \\
\hline $\begin{array}{l}\text { Sea water } \\
\text { from Tsuna- } \\
\text { shirazu cove } \\
\text { Surface }\end{array}$ & $\begin{array}{l}93.0 \\
92.0 \\
89.0\end{array}$ & $\begin{array}{r}9.0 \\
8.5 \\
13.0\end{array}$ & $\begin{array}{l}91.0 \\
91.5 \\
87.0\end{array}$ & $\begin{array}{l}0 \\
0 \\
0\end{array}$ & 4.0 & 96.0 & 0 & & 1 \\
\hline Bottom (5) & $\begin{array}{l}89.0 \\
86.5 \\
83.5\end{array}$ & $\begin{array}{l}11.0 \\
13.5 \\
18.0\end{array}$ & $\begin{array}{l}87.5 \\
85.5 \\
81.0\end{array}$ & $\begin{array}{l}1.5 \\
1.0 \\
1.0\end{array}$ & 7.0 & 93.0 & 0 & & 1 \\
\hline
\end{tabular}

Table 3. Results of the Aug. 6 experiment with eggs of Anthocidaris crassispina. Wind; 0 . Test water temperature; $29^{\circ} \mathrm{C} .3 \mathrm{hrs}$. old eggs

\begin{tabular}{|c|c|c|c|c|c|c|c|c|c|}
\hline \multirow[b]{2}{*}{$\begin{array}{l}\text { Location } \\
\text { (depth) }\end{array}$} & \multirow{2}{*}{\begin{tabular}{|l} 
Fertiliz. \\
membrane \\
formation
\end{tabular}} & \multicolumn{3}{|c|}{ First cleavage (50 min.) } & \multicolumn{3}{|c|}{ Gastrulation (15 hrs.) } & \multirow{2}{*}{\begin{tabular}{|c|}
$\begin{array}{c}\text { Other } \\
\text { notes }\end{array}$ \\
ab- \\
normal \\
develop.
\end{tabular}} & \multirow{2}{*}{$\begin{array}{c}\text { Degree } \\
\text { of in- } \\
\text { hibitory } \\
\text { effect }\end{array}$} \\
\hline & & 1 cell & $\begin{array}{c}2 \text { cell } \\
\text { (normal) }\end{array}$ & $\mid \begin{array}{c}\text { multi- } \\
\text { cell(poly- } \\
\text { spermy) }\end{array}$ & $\mid$\begin{tabular}{c|} 
perma- \\
nent \\
blastula
\end{tabular} & $\begin{array}{l}\text { gastrula } \\
\text { (normal) }\end{array}$ & $\begin{array}{c}\text { exo- } \\
\text { gastrula }\end{array}$ & & \\
\hline $\begin{array}{l}\text { Running }(\mathrm{m}) \\
\text { sea water of } \\
\text { Laboratory }\end{array}$ & $\begin{array}{l}97.5 \% \\
99.0 \\
99.5\end{array}$ & $\begin{array}{l}3.0 \% \\
1.5 \\
1.0\end{array}$ & $\begin{array}{l}96.5 \% \\
98.5 \\
99.0\end{array}$ & $\begin{array}{l}0.5 \% \\
0 \\
0\end{array}$ & $0 \%$ & $100^{\%}$ & 0 & & 0 \\
\hline $\begin{array}{l}\text { Water from } \\
\text { open sea side } \\
\text { of Hatake- } \\
\text { jima Surface }\end{array}$ & $\begin{array}{l}97.5 \\
97.0 \\
96.0\end{array}$ & $\begin{array}{l}3.5 \\
4.0 \\
3.5\end{array}$ & $\begin{array}{l}96.0 \\
95.0 \\
95.5\end{array}$ & $\begin{array}{l}0.5 \\
1.0 \\
1.0\end{array}$ & 1.0 & 99.0 & 0 & & 0 \\
\hline Bottom (25) & $\begin{array}{l}96.0 \\
97.0 \\
96.5\end{array}$ & $\begin{array}{l}4.5 \\
4.5 \\
5.0\end{array}$ & $\begin{array}{l}94.5 \\
95.0 \\
94.0\end{array}$ & $\begin{array}{l}1.0 \\
0.5 \\
1.0\end{array}$ & 0.5 & 99.5 & 0 & & 0 \\
\hline $\begin{array}{l}\text { Water from } \\
\text { land side of } \\
\text { Hatakejima } \\
\text { Surface }\end{array}$ & $\begin{array}{l}89.5 \\
88.5 \\
90.0\end{array}$ & $\begin{array}{l}12.5 \\
12.0 \\
11.0\end{array}$ & $\begin{array}{l}84.0 \\
85.0 \\
84.5\end{array}$ & $\begin{array}{l}3.5 \\
3.0 \\
4.5\end{array}$ & 1.5 & 98.5 & 0 & & 1 \\
\hline Bottom (27) & $\begin{array}{l}80.5 \\
83.5 \\
79.0\end{array}$ & $\begin{array}{l}23.0 \\
21.5 \\
22.0\end{array}$ & $\begin{array}{l}70.5 \\
73.5 \\
72.0\end{array}$ & $\begin{array}{l}6.5 \\
\mathbf{5 . 0} \\
6.0\end{array}$ & 1.5 & 98.5 & 0 & & 2 \\
\hline $\begin{array}{l}\text { Sea water } \\
\text { from Tsuna- } \\
\text { shirazu cove } \\
\text { Suraface }\end{array}$ & $\begin{array}{l}80.5 \\
76.5 \\
79.0\end{array}$ & $\begin{array}{l}21.5 \\
23.0 \\
22.5\end{array}$ & $\begin{array}{l}73.5 \\
71.0 \\
70.0\end{array}$ & $\begin{array}{l}5.0 \\
6.0 \\
7.5\end{array}$ & 7.5 & 92.5 & 0 & & 2 \\
\hline Bottom (5) & $\begin{array}{l}78.0 \\
76.0 \\
71.5\end{array}$ & $\begin{array}{l}25.0 \\
24.5 \\
27.0\end{array}$ & $\begin{array}{l}68.5 \\
70.0 \\
64.5\end{array}$ & $\begin{array}{l}6.5 \\
5.5 \\
8.5\end{array}$ & 6.5 & 93.5 & 0 & & 3 \\
\hline
\end{tabular}


Table 4. Results of the Sept. 14 experiment with eggs of Anthocidaris crassispina. Wind; 0 . Test water temperature; $25^{\circ} \mathrm{C}$. $3 \mathrm{hrs}$. old eggs

\begin{tabular}{|c|c|c|c|c|c|c|c|c|c|}
\hline \multirow{2}{*}{$\begin{array}{c}\text { Location } \\
\text { (depth) }\end{array}$} & \multirow{2}{*}{\begin{tabular}{c|} 
Fertiliz. \\
membrane \\
formation
\end{tabular}} & \multicolumn{3}{|c|}{ First cleavage $(60 \mathrm{~min})}$. & \multicolumn{3}{|c|}{ Gastrulation (18 hrs.) } & \multirow{2}{*}{\begin{tabular}{|c|}
$\begin{array}{c}\text { Other } \\
\text { notes }\end{array}$ \\
ab- \\
normal \\
develop.
\end{tabular}} & \multirow{2}{*}{$\begin{array}{c}\text { Degree } \\
\text { of in- } \\
\text { hibitory } \\
\text { effect }\end{array}$} \\
\hline & & 1 cell & $\begin{array}{c}2 \text { cell } \\
\text { (normal) }\end{array}$ & $\begin{array}{c}\text { multi- } \\
\text { cell(poly- } \\
\text { spermy) }\end{array}$ & $\begin{array}{c}\text { perma- } \\
\text { nent } \\
\text { blastula }\end{array}$ & $\begin{array}{r}\text { gastrula } \\
\text { (normal) }\end{array}$ & $\begin{array}{c}\text { exo- } \\
\text { gastrula }\end{array}$ & & \\
\hline $\begin{array}{l}\text { Running }(\mathrm{m}) \\
\text { sea water of } \\
\text { Laboratory }\end{array}$ & $\begin{array}{l}98.5 \% \\
99.0 \\
98.5\end{array}$ & $\begin{array}{l}2.0 \% \\
1.0 \\
1.5\end{array}$ & $\begin{array}{l}98.0 \% \\
99.0 \\
98.5\end{array}$ & \begin{tabular}{ll|}
0 & $\%$ \\
0 & \\
0 & \\
\end{tabular} & $0.5^{\%}$ & $99.5^{\%}$ & $0^{2 \%}$ & & $\mathbf{0}$ \\
\hline $\begin{array}{l}\text { Water from } \\
\text { open sea side } \\
\text { of Hatake- } \\
\text { jima Suface }\end{array}$ & $\begin{array}{l}99.0 \\
99.5 \\
98.0\end{array}$ & $\begin{array}{l}1.5 \\
0.5 \\
3.0\end{array}$ & $\begin{array}{l}98.5 \\
99.5 \\
97.0\end{array}$ & $\begin{array}{l}0 \\
0 \\
0\end{array}$ & 0.5 & 99.5 & 0 & & 0 \\
\hline $\begin{array}{l}\text { Water from } \\
\text { land side of } \\
\text { Hatakejima } \\
\text { Surface }\end{array}$ & $\begin{array}{l}90.5 \\
91.0 \\
88.5\end{array}$ & $\begin{array}{l}11.5 \\
10.5 \\
14.0\end{array}$ & $\begin{array}{l}88.5 \\
89.5 \\
86.0\end{array}$ & $\begin{array}{l}0 \\
0 \\
0\end{array}$ & 1.5 & 98.5 & 0 & & 1 \\
\hline $\begin{array}{l}\text { Sea swater } \\
\text { from Tsuna- } \\
\text { shirazu cove } \\
\text { Surface }\end{array}$ & $\begin{array}{l}84.0 \\
85.5 \\
84.0\end{array}$ & $\begin{array}{l}16.0 \\
15.0 \\
18.0\end{array}$ & $\begin{array}{l}83.5 \\
85.0 \\
81.5\end{array}$ & $\begin{array}{l}0.5 \\
0 \\
0.5\end{array}$ & 5.5 & 94.5 & 0 & & 1 \\
\hline
\end{tabular}

Table 5. Results of the Nov. 27 experiment with eggs of Pseudocentrotus depressus. Wind; 0 . Test water temperature; $20^{\circ} \mathrm{C}$. $8 \mathrm{hrs}$. old eggs

\begin{tabular}{|c|c|c|c|c|c|c|c|c|c|}
\hline \multirow{2}{*}{$\begin{array}{c}\text { Location } \\
\text { (depth) }\end{array}$} & \multirow{2}{*}{$\begin{array}{l}\text { Fertiliz. } \\
\text { membrane } \\
\text { formation }\end{array}$} & \multicolumn{3}{|c|}{ First cleavage (80 min.) } & \multicolumn{3}{|c|}{ Gastrulation (20 hrs.) } & \multirow{2}{*}{\begin{tabular}{c|}
$\begin{array}{c}\text { Other } \\
\text { notes }\end{array}$ \\
ab- \\
normal \\
develop.
\end{tabular}} & \multirow{2}{*}{$\begin{array}{c}\text { Degree } \\
\text { of in- } \\
\text { hibitory } \\
\text { effect }\end{array}$} \\
\hline & & 1 cell & $\begin{array}{c}2 \text { cell } \\
\text { (normal) }\end{array}$ & $\left|\begin{array}{c}\text { multi- } \\
\text { cell(poly- } \\
\text { spermy) }\end{array}\right|$ & $\begin{array}{c}\text { perma- } \\
\text { nent } \\
\text { blastula }\end{array}$ & \begin{tabular}{|} 
gastrula \\
(normal)
\end{tabular} & $\begin{array}{c}\text { exo- } \\
\text { gastrula }\end{array}$ & & \\
\hline \multirow{2}{*}{$\begin{array}{l}\text { Running }(\mathrm{m}) \\
\text { sea water of } \\
\text { Laboratory }\end{array}$} & $98.5 \%$ & $1.0 \%$ & $98.0^{\%}$ & $1.0 \%$ & $\%$ & $\%$ & $\%$ & & \\
\hline & 99.0 & 1.0 & 98.5 & 0.5 & 1.5 & 98.0 & 0 & & 0 \\
\hline \multirow{2}{*}{$\begin{array}{l}\text { Water from } \\
\text { open sea side } \\
\text { of Hatake- } \\
\text { jima Surface }\end{array}$} & 99.0 & 1.0 & 98.5 & 0.5 & \multirow{2}{*}{0} & \multirow{2}{*}{100} & \multirow[b]{2}{*}{0} & & \multirow{2}{*}{0} \\
\hline & 99.5 & 0.5 & 99.0 & 0.5 & & & & & \\
\hline \multirow{2}{*}{$\begin{array}{l}\text { Water from } \\
\text { land side of } \\
\text { Hatakejima } \\
\text { Surface }\end{array}$} & 98.5 & 1.5 & 97.5 & 1.0 & \multirow{2}{*}{1.0} & \multirow{2}{*}{99.0} & \multirow{2}{*}{0} & & \multirow{2}{*}{0} \\
\hline & 98.5 & 1.5 & 98.0 & 0.5 & & & & & \\
\hline \multirow{2}{*}{ Bottom (27) } & 97.5 & 2.0 & 96.5 & 1.5 & \multirow{2}{*}{1.5} & \multirow{2}{*}{98.5} & \multirow{2}{*}{0} & & \multirow{2}{*}{0} \\
\hline & 97.5 & 1.5 & 97.0 & 1.5 & & & & & \\
\hline \multirow{2}{*}{$\begin{array}{l}\text { Sea water } \\
\text { from Tsuna- } \\
\text { shirazu cove } \\
\text { Surface }\end{array}$} & 97.0 & 3.0 & 95.0 & 2.0 & \multirow{2}{*}{0.5} & \multirow{2}{*}{99.5} & \multirow{2}{*}{0} & & \multirow{2}{*}{0} \\
\hline & 97.5 & 2.0 & 96.0 & 2.0 & & & & & \\
\hline \multirow{2}{*}{ Bottom (5) } & 95.5 & 3.5 & 94.0 & 2.5 & \multirow{2}{*}{1.0} & \multirow{2}{*}{99.0} & \multirow{2}{*}{0} & & \multirow{2}{*}{1} \\
\hline & 96.0 & 2.0 & 95.0 & 3.0 & & & & & \\
\hline
\end{tabular}

aging of 3-8 hours; in Degree of inhibitory effect, 0 shows no inhibition, 1 a slight inhibition, 2 a weak and 3 a moderate inhibition by the sea water tested (see Publ. Seto Mar. Biol. Lab., XXI (5/6), p. 391, 8 tables, 1974). 